\title{
La fundamental convergencia teórica entre el pensamiento económico de Adam Smith y el de los pioneros de la sociología británica*
}

\section{The fundamental theoretical convergence between Adam Smith's}

economic thinking and that of the pioneers of British sociology

Sagar Hernández Chuliá**

Recibido: 14 de enero de 2017

Revisado: 10 de febrero de 2017

Aprobado: 6 de febrero de 2017

* Este artículo constituye una adaptación del epígrafe 1.2 "Relación de la escuela clásica de economía con los pioneros y fundadores en sociología" de mi tesis doctoral titulada Principales puntos de convergencia entre las escuelas hegemónicas de teoría económica y sociológica desde la Ilustración escocesa hasta nuestros días.

** Doctor en Sociología por la Universidad Complutense de Madrid. Departamento de Sociología V (Teoría Sociológica). Programa de doctorado: "Teoría Sociológica: Cultura, Conocimiento y Comunicación”. Madrid. (España). Correo electrónico: sagarhch@gmail.com 


\section{Resumen}

Con esta investigación pretendemos demostrar la fundamental convergencia teórica existente entre el pensamiento económico de Adam Smith, autor que para muchos economistas actuales es el fundador de su disciplina, y el de los pioneros de la sociología británica: Adam Ferguson y John Millar. Para tal fin, agrupamos y comparamos los postulados defendidos por estos tres autores en torno a seis categorías: teleología, epistemología, metodología, agente individual, organizaciones e instituciones. A la vista de los resultados, concluimos que dicha convergencia teórica no solo existe, sino que, además, puede explicarse atendiendo a su común origen en la obra de David Hume.

Palabras clave: Teoría económica, teoría sociológica, pioneros de la sociología, Adam Smith.

Glasificación JEL: A12, B12, N01, Y80.

\section{Abstract}

With this research, we want to demonstrate the fundamental theoretical convergence between the economic thought of Adam Smith, author who for many economists of today is the founder of their discipline, and of the pioneers of the British sociology: Adam Ferguson and John Millar. To this end, we group and compare the principles defended by these three authors around six categories: teleology, epistemology, methodology, individual agents, organizations and institutions. In view of the results, we conclude that this theoretical convergence, not only exists, but also can be explained according to their common origin in the work of David Hume.

Keywords: Economic theory, sociological theory, pioneers of the sociology, Adam Smith.

Classification JEL: A12, B12, N01, Y80. 


\section{Introducción}

El profesor Emilio Lamo de Espinosa (2001) propone una aproximación a la historia del pensamiento sociológico que divide la sociología en cinco generaciones. Según dicha taxonomía, los pioneros de esta disciplina serían "los nominalistas escoceses del XVIII o Montesquieu" (Lamo, 2001, p. 30). Por otra parte, entre las aportaciones realizadas por dichos "nominalistas escoceses del siglo XVIII" al pensamiento sociológico, tradicionalmente se han destacado las obras de Adam Ferguson (Barnes, 1917; Benton, 1978; Brewer, 2007; Bryson, 1939; Lehmann, 1930; MacRae, 1969; Swingewood, 1970) y John Millar (Lehmann, 1960; Swingewood, 1970). Para demostrar la fundamental convergencia teórica existente entre las contribuciones procedentes de estos dos últimos y las de Adam Smith1, pensador que para muchos economistas actuales es considerado como el fundador de su disciplina, agrupamos y comparamos sus respectivos postulados en torno a seis categorías principales. Las tres primeras (teleología, epistemología y metodología) proceden de la clásica taxonomía habermasiana relativa a los tipos de conocimiento e interés2 (Habermas, 1968) y nos permiten cotejar cualesquiera disciplinas científicas; mientras que las tres últimas (agente individual, organizaciones e instituciones) aluden a tres conceptos teóricos que, al menos en este momento histórico, resultan centrales para ambas esferas de pensamiento (economía y sociología).

\section{Teleología}

La demostración de nuestra hipótesis supone partir de la afirmación de que tanto Adam Smith como los pioneros de la sociología británica priorizan el interés práctico de comprensión sobre otras formas de interés cognoscitivo. Habermas (1968, p. 42) parte de considerar que "la ciencia tiende a engañarse a sí misma acerca de intereses fundamentales, a los que no solo debe su fuerza impulsora, sino las propias condiciones de la objetividad posible". Según este autor, podemos identificar tres tipos de acciones básicas: trabajo, lenguaje e interacción social, a cada una de las cuales le corresponde, respectivamente, un tipo de interés: técnico, práctico de comprensión y emancipatorio. Nosotros consideramos que estas son las tres posibles formas que, efectivamente, puede presentar nuestra primera categoría: la teleológica. Sin embargo, Habermas añade que cada una de ellas alude a un cierto tipo de ciencia (empírico-analítica, histórico-hermenéutica o crítica), mientras que nosotros defendemos que pueden presentarse en cualquier disciplina científica, incluidas, claro está, la economía y la sociología. Por lo tanto,

1 En este sentido, el propio Albion Small (1907) llega a señalar la posibilidad de realizar una lectura sociológica de los textos de Adam Smith.

2 Una taxonomía que, aunque el propio Habermas pueda considerar superada por su teoría de la acción comunicativa, y pese a que no se elabore con este propósito explícito, resulta de gran utilidad para este tipo de investigaciones. 
nuestro objetivo en esta primera categoría debe limitarse a señalar la prioridad que cada uno de estos autores atribuye a un determinado tipo de interés cognoscitivo en su obra.

Tanto Una investigación sobre la naturaleza y causas de la riqueza de las naciones (Smith, [1776] 1904) como Lecciones de jurisprudencia (Smith, [1762-63] 1896) son, en palabras del propio Adam Smith, tan solo partes de una obra más amplia de carácter histórico referida a "los principios generales del derecho y del gobierno, y de las diferentes revoluciones que en estos se han producido en las diferentes épocas y períodos de la sociedad" (Smith, [1759] 1853, p. 503). Como podemos comprobar, el interés práctico de comprensión se impone sobre las consideraciones técnicas o emancipatorias. Sin embargo, resultaría injusto considerar que el pensamiento de este autor se limita a analizar el funcionamiento del derecho o del gobierno. En este sentido, por ejemplo, en La riqueza de las naciones (Smith, [1776] 1904) podemos encontrar gran cantidad de pasajes con recomendaciones relativas a la política económica (interés técnico), y en La teoría de los sentimientos morales (Smith, [1759] 1853), especialmente en el capítulo VII, no son escasas las alusiones a una recuperación de la doctrina estoica clásica que funcione a modo de mecanismo preventivo frente a conductas antisociales (interés emancipatorio).

Por otro lado, el texto más conocido de Adam Ferguson ([1767] 1782), Ensayo sobre la historia de la sociedad civil, es, antes que nada, un pormenorizado estudio sobre cómo han ido evolucionando las diferentes sociedades a lo largo de la historia (interés práctico de comprensión). No obstante, también puede leerse como una crítica a la forma comercial que estas han adoptado en su tiempo, ya que, según el propio autor, dicha configuración promueve el abandono de virtudes cívicas tan relevantes como el patriotismo o la participación política (interés emancipatorio). Esta doble lectura se apoya en dos afirmaciones de Ferguson. Por una parte, señala -inclinándose por la primera aproximación- que "al igual que los matemáticos (...) nunca piensan en medir, [nosotros] leemos las sociedades, pero no nos proponemos actuar con los hombres" (Ferguson, ([1767] 1782, p. 49); mientras que, por otra, reconoce que "es imposible mantener el tono especulativo siempre. Es imposible no sentir a veces que vivimos entre los hombres" (Ferguson, [1767] 1782, p. 51).

Por su parte, John Millar ([1771] 2006), en Observaciones sobre la distinción de rangos en la sociedad, pretende dar cuenta de la evolución histórica que han sufrido las relaciones sociales atendiendo a sus correspondientes sistemas económicos (interés práctico de comprensión). Sin embargo, no renuncia tampoco a introducir sugerencias concretas a fin de reducir las consecuencias perversas a las que, según él, puede dar lugar la desigualdad social (interés emancipatorio).

En cualquier caso, y sin pretenderlo estos autores, el principal motivo que explica la gran importancia que han ido adquiriendo sus respectivas obras con paso del tiempo remite a su capacidad para dotar de legitimidad a dos nuevos campos de estudio: la economía y 
la sociología. No obstante, dicho proceso ha favorecido también la introducción en ambas esferas de conceptos procedentes de la filosofía moral. Un ejemplo paradigmático en este sentido lo encontramos en una obra que ha sido considerada durante largo tiempo como el texto fundacional de la economía moderna: Una indagación sobre la naturaleza y las causas de la riqueza de las naciones (Smith, [1776] 1904). Este escrito resulta ser, en realidad, una aplicación práctica de la filosofía moral expuesta diecisiete años antes por Adam Smith ([1759] 1853) en su Teoría de los sentimientos morales. Sin embargo, tal hecho no debe sorprendernos, ya que este autor impartió docencia precisamente sobre dicha materia en la Universidad de Glasgow entre 1751 y 1763.

Este proceso de arrastre de conceptos desde la filosofia moral también se ve reflejado en Ensayos sobre la historia de la sociedad civil, de Adam Ferguson ([1767] 1782). Como acabamos de señalar, esta obra puede leerse no solo como uno de los primeros trabajos sociológicos, sino como un manual de virtudes estoicas. No por casualidad Ferguson ocupó la plaza de profesor de Filosofía Moral desde 1764 hasta 1785, aunque en su caso, en la Universidad de Edimburgo.

Finalmente, John Millar ([1771] 2006), en su texto sobre El origen de la distinción de rangos, argumenta que, conforme la sociedad se enriquece, aumenta la libertad de sus ciudadanos hasta el punto de considerar que la esclavitud no solo es poco eficiente en el ámbito estrictamente económico, sino que además resulta moralmente perversa. Por cierto, este mismo autor también defiende en otra de sus obras (Millar, [1803] 2006) la idea de que la libertad puede emerger como una consecuencia no prevista del enfrentamiento entre tiranos. Así, ambos ejemplos nos sirven para ilustrar la presencia en sus escritos de conceptos originarios de la filosofía moral.

\section{Epistemología}

Nuestra categoría epistemológica remite al concepto habermasiano de reglas lógicometódicas. Como en la categoría precedente, también aquí identificamos tres tipos principales. Por un lado, nos encontramos con las reglas hipotético-deductivas, que provienen del interés técnico y "permiten deducir hipótesis legiformes dotadas de contenido empírico. (...) Pueden interpretarse como enunciados acerca de la covarianza de magnitudes observables" (Habermas, 1968, p. 39). Por otro lado, en las reglas histórico-hermenéuticas, nacidas del interés práctico de comprensión, "es la comprensión del sentido y no la observación la que nos permite acceder a los hechos" (Habermas, 1968, p. 40). Finalmente, el interés emancipatorio se sirve de la autorreflexión, de tal modo que esta "libera al sujeto de su dependencia de poderes hipostasiados" (Habermas, 1968, p. 41). No obstante, aunque para efectos estrictamente heurísticos aceptamos esta taxonomía, defendemos que estas tres formas pueden combinarse para dar lugar a configuraciones 
híbridas, y aquí nos distanciamos parcialmente de la doctrina original propuesta por Habermas.

Acabamos de afirmar la prioridad que Adam Smith y los pioneros de la sociología británica otorgan al interés práctico de comprensión. Según el modelo original de Habermas (1968), a este le corresponde el desarrollo de reglas histórico-hermenéuticas. Pero, ¿es realmente así? Antes que nada, debemos comenzar atendiendo a la diferencia que establece David Hume ([1739] 1896) -autor que, por cierto, también manifiesta un interés prioritario práctico de comprensión- entre dos clases de razonamiento: relaciones de hechos y relaciones de ideas. Las primeras remiten al proceso inductivo basado en la observación. En esta fase, el investigador genera vínculos entre distintos fenómenos observados y en su mente brota una idea. Las segundas, lógica y temporalmente posteriores, consisten en el establecimiento de conexiones entre dicha idea y otras de su especie mediante procedimientos deductivos basados en la comprensión del sentido. Esta forma epistemológica supone, en definitiva, la instauración de un nuevo tipo de reglas de carácter híbrido que combina características hipotético-deductivas (primer momento) e histórico-hermenéuticas (segundo momento).

Dicha doctrina es seguida muy de cerca por Adam Smith ${ }^{3}$. Así, este autor afirma inequívocamente:

Es evidente que la mente se complace en observar las semejanzas que se pueden descubrir entre diferentes objetos. Es por medio de este tipo de observaciones que se esfuerza por arreglar y sistematizar [methodise] todas sus ideas y reducirlas a clases y categorías [assortments] adecuadas. (Smith, 1795, p. 10).

Smith además introduce el componente hermenéutico del conocimiento al remitir el origen de dicho proceso a la capacidad de asombro que demuestra el ser humano ante "los objetos extraordinarios y no comunes, ante los fenómenos más raros de la naturaleza" (Smith, 1795, p. 3). Esto despierta "naturalmente (...) la curiosidad de la humanidad por investigar sus causas" (Smith, [1776] 1904, p. 256 II). De ese modo, por ejemplo, cuando dos objetos suelen presentarse habitualmente vinculados precediendo el uno al otro, "llegan a estar tan conectados entre sí en nuestra fantasía, que la idea de uno parece introducir la del otro" (Smith, 1795, p. 14). Por cierto, este componente hermenéutico, que según Smith (1795, pp. 1-29) se apoya en la admiración, el asombro y la sorpresa del investigador, también es incorporado, aunque sin citar la fuente, por John Millar ([1803] 2006, pp. 729-730).

En esta misma línea se manifiesta Adam Ferguson al afirmar:

Los objetos que nos rodean, además de sus apariciones por separado, presentan relaciones mutuas. Sugieren, cuando los comparamos, lo que no ocurriría cuando se consideran

3 Sobre la posición epistemológica de Smith, véase Skinner (1972). 
aparte. Tienen efectos e influencias mutuas y exhiben, en circunstancias similares, operaciones equivalentes y consecuencias uniformes. (Ferguson, [1767] 1782, p. 44).

De este modo, según Ferguson el objeto de toda ciencia consiste en "recopilar una multiplicidad de datos particulares bajo pensamientos [heads] generales y remitir varias operaciones a su principio común" (Ferguson, [1767] 1782, p. 44). No obstante, tal forma de proceder no resulta privativa de los científicos. Todo individuo parte "de la observación y la experiencia para encontrar los puntos de vista generales bajo los cuales puede considerar sus objetos" (Ferguson, [1767] 1782, p. 45).

Por otro lado, John Millar ([1803] 2006), en Una visión histórica del Gobierno inglés, pretende "ilustrar la historia natural de la humanidad" (Millar, [1803] 2006, p. 89) y señala, por una parte, "las mejoras más obvias y comunes que surgen gradualmente en sociedad" (Millar, [1803] 2006, p. 90) y, mientras que, por otra, muestra "su influencia sobre las costumbres, las leyes y el gobierno de un pueblo" (Millar, [1803] 2006, p. 90). Así, comienza señalando la dificultad que entraña obtener "materiales adecuados para especulaciones de esta naturaleza" (Millar, [1803] 2006, p. 90) o, como también los denomina, "hechos" (Millar, [1803] 2006, p. 90) de los cuales valerse en su investigación. Estos, que hacen las veces de fenómenos observados, deben venir avalados por diferentes fuentes a fin de reducir el riesgo de introducir distorsiones interesadas. Eso sí, una vez disponemos de ellos, podemos "añadir las razones de esas costumbres particulares que se han reportado de manera uniforme" (Millar, [1803] 2006, p. 90), es decir, somos capaces de ponerlos en relación unos con otros y establecer, de este modo, vínculos causales entre ideas.

De este modo, el tipo metodológico híbrido que postula Hume da lugar al establecimiento de modelos teóricos, o "sistemas"4, en los que convergen la observación y la comprensión del sentido, y que operan de un modo muy similar al de las máquinas:

Una máquina es un pequeño sistema creado para llevar a cabo y conectar entre sí, en la realidad, distintos movimientos y efectos (...). Un sistema es una máquina imaginaria inventada para conectar en la fantasía diferentes movimientos y efectos que ya operan en la realidad. (Smith, 1795, p. 44).

Un buen ejemplo en este sentido es la división que Smith establece entre cuatro tipos de sociedades humanas: pueblos de cazadores, pueblos de pastores, naciones agrícolas y feudales y países comerciales. Por cierto, la importancia que este autor atribuye al componente histórico también es la que explica que en su principal obra podamos encontrar un análisis sobre las fluctuaciones en el valor de la plata durante los últimos cuatro siglos (Smith, [1776] 1904, pp. 177-216 I), una historia económica europea desde la caída del Imperio Romano (Smith, [1776] 1904, pp. 360-370 I), un estudio de las políticas

4 Sobre el concepto de "sistema" en Smtih, véase Skinner (1976). 
comerciales y coloniales de las naciones europeas (Smith, [1776] 1904, pp. 396-417 I) o diversas reflexiones sobre los diferentes métodos de defensa y administración de justicia en sociedades "primitivas" (Smith, [1776] 1904, pp. 186-214 II).

Por su parte, en Observaciones sobre la distinción de rangos en la sociedad, Millar ([1771] 2006) introduce la misma taxonomía de la que se vale Smith para agrupar las sociedades humanas, mientras que en Una visión histórica del Gobierno inglés (Millar, [1803] 2006) analiza la evolución de las libertades civiles y políticas en este país en tres períodos distintos. Por cierto, en esta última obra también da entrada a la metáfora "maquinista" al afirmar que "en todo sistema de leyes o gobierno, las diferentes partes presentan una íntima conexión entre sî" (Millar, [1803] 2006, p. 285). De ello se infiere, según él, que "es peligroso manipular la máquina" (Millar, [1803] 2006, p. 285), ya que "la alteración violenta de cualquier parte solo puede destruir la regularidad de sus movimientos y producir el mayor desorden y confusión" (Millar, [1803] 2006, p. 285).

Finalmente, Ferguson se decanta por dividir las sociedades humanas en tres tipos: rudas, bárbaras y educadas. Así, como Smith y Millar, procede mediante un método analítico que podemos denominar "historia conjetural", que se caracteriza por elaborar reconstrucciones racionales de la historia partiendo de la uniformidad de la naturaleza humana y del establecimiento de causas probables. En este sentido, el propio Dugald Stewart (1795, p. xl), sucesor de Ferguson en Edimburgo y autor que bautiza esta nueva forma de investigación, destaca cómo la Disertación sobre el origen de los lenguajes de Smith constituye un destacado ejemplo de dicha doctrina. En este mismo sentido, no debemos perder de vista cómo Millar ([1803] 2006, p. 446), al estudiar el Gobierno de Escocia desde que los romanos abandonaron Gran Bretaña hasta el reinado de Malcolm II, afirma que "durante este primer período, poco se sabe con certeza. Debemos satisfacernos con una delineación basada en una conjetura probable".

\section{Metodología}

La metodología constituye la tercera categoría a la que atendemos. Según Habermas (1968), podemos identificar tres tipos principales: empírico-analítica, lógico-deductiva y dialéctica. Cada una de ellas proviene, respectivamente, de las reglas hipotético-deductivas, histórico-hermenéuticas y autorreflexivas. La primera categoría opera aislando los elementos del fenómeno considerados pertinentes, para, a partir de ahí, analizarlos por separado, e incorpora tanto el método experimental (de laboratorio) como el de medición (empírico-matemático). Por su parte, la segunda categoría aplica principios generales previamente establecidos a casos particulares, mediante un encadenamiento de razonamientos lógicos de los que se deduce una conclusión. Finalmente, la metodología dialéctica se caracteriza por enfatizar la concepción dinámica de la historia desde 
una perspectiva marxista. Estos tres tipos se corresponden con las principales formas metodológicas de las que nos valemos a la hora de comparar las aportaciones teóricas procedentes de estos autores. Ahora bien, como en los apartados precedentes, también aquí contemplamos, a diferencia de Habermas (1968), la posibilidad de identificar formas intermedias.

Acabamos de afirmar que tanto Adam Smith como los pioneros de la sociología británica defienden una forma epistemológica híbrida de reglas hipotético-deductivas e históricohermenéuticas. No obstante, cabe preguntarse qué consecuencias supone dicha decisión en el ámbito metodológico. Pues bien, estos autores trasladan, sin modificación alguna, su apuesta epistemológica al ámbito metodológico. Así, comienzan identificando en un primer momento, y con base en su experiencia - es decir, de manera empírico-analíti$\mathrm{ca}^{-}$, aquellos factores que consideran más relevantes, para posteriormente establecerlos como principios generales de los cuales deducir conclusiones mediante procedimientos lógicos - a la manera lógico-deductiva- ${ }^{-}$. Es decir, nos encontramos en presencia de una metodología híbrida de carácter secuencial.

En este sentido, según Adam Smith ([1776] 1904, p. 256 II), el conocimiento humano puede dividirse en tres grandes categorías: la física (o filosofía natural), la ética (o filosofía moral) y la lógica. La segunda, objeto prioritario de sus investigaciones, se ocupa, con ayuda de la tercera, del estudio de "las máximas de la vida en común dispuestas en un orden metódico y conectadas entre sí por ciertos principios comunes" (Smith, [1776] 1904, p. 257 II). A su vez, dichas máximas remiten a conceptos tales como "ética”, "justicia" y "economía", objetos de estudio centrales, respectivamente, de sus textos La teoría de los sentimientos morales, Lecturas sobre jurisprudencia y La riqueza de las naciones.

De este modo, por ejemplo, la metodología de la que se vale en este último texto pretende superar las prácticas de aquellos autores previos que, como Esopo, se han limitado a recoger las tradicionales máximas de prudencia y moralidad sin "intentar organizarlas en un claro orden metódico y mucho menos conectarlas entre sí [por medio] de uno o varios principios generales de los que todas ellas sean deducibles" (Smith, [1776] 1904, p. 257 II). Para superar dicho defecto, y basándose en su experiencia, Smith establece como principio ordenador de su sistema "el esfuerzo natural de cada individuo por mejorar su propia condición” (Smith, [1776] 1904, p. 43 II), un empeño que califica de "universal, continuo e ininterrumpido" (Smith, [1776] 1904, pp. 327-328 I). Por cierto, el destacado papel que este autor otorga a la experiencia como instrumento metodológico también es el que explica la presencia en este texto de numerosas estadísticas (método de medición empírico-matemático) que operan a modo de confirmación de sus afirmaciones teóricas.

Por otra parte, y con respecto al papel que desempeña la lógica en este proyecto, Smith ([1776] 1904, p. 257 II) nos recuerda como "los autores de cada sistema de filosofía 
natural y moral se esfuerzan (...) por exponer la debilidad de los argumentos aducidos para apoyar sistemas alternativos" haciendo uso de la lógica o "ciencia de los principios generales del buen y mal razonamiento". No debemos perder de vista que Smith, aunque profesor de Filosofía Moral en la Universidad de Glasgow durante muchos años, comienza su labor docente enseñando lógica.

En este mismo sentido, Ferguson ([1767] 1782, p. 3), al referirse al "deseo [de algunos autores] de sentar las bases de un sistema", llama la atención sobre el error que cometen al seleccionar, "entre las diversas cualidades que la humanidad posee" (Ferguson, [1767] 1782, p. 3), ciertas características con el fin de "establecer una teoría” (Ferguson, [1767] 1782 , p. 3), pasando por alto "lo que siempre ha aparecido dentro del alcance de nuestra propia observación y en los registros de la historia” (Ferguson, [1767] 1782, p. 3). Así, dicha forma de proceder, alejada de la experiencia, ha dado lugar a "numerosas consultas infructuosas y ha generado muchas suposiciones salvajes" (Ferguson, [1767] 1782, p. 3).

Frente a esta estrategia, Ferguson ([1767] 1782, p. 107) defiende que, "a fin de tener un conocimiento general e integral de la totalidad, debemos (...) pasar por alto muchos detalles y singularidades (...) y fijar nuestra atención en algunas cuestiones". Una vez asentadas estas cuestiones, "hemos adquirido un conocimiento que, aunque no reemplaza la necesidad de experiencia, puede servir para guiar nuestras investigaciones" (Ferguson, [1767] 1782, p. 107). De este modo, podemos "establecer un orden y un método a la hora de disponer los datos que se dan a nuestra observación" (Ferguson, [1767] 1782, p. 107). Es decir, esta forma de proceder implica dos fases: una primera, en la que el investigador recurre a la experiencia, y una segunda, en la que se vale de mecanismos lógico-deductivos.

Por otro lado, ya hemos señalado cómo Millar se vale de "hechos" procedentes de fuentes de segunda mano cuya validez descansa en la reiteración de testimonios. Ahora bien, una vez certificada esta, debemos proceder, al modo de Smith, estableciendo un principio organizador del sistema que los ordene y otorgue sentido. Dicho principio no es otro que "la disposición y capacidad del hombre para mejorar su propia condición" (Millar, [1803] 2006, p. 84). Este postulado, según Millar, no deriva de "abstractas teorías metafísicas" (Millar, [1803] 2006, p. 285), sino que se establece mediante lo que él denomina, al más puro estilo empírico-analítico, "experimentos reales" (Millar, [1803] 2006, p. 285). Es gracias a este método que "la naturaleza humana se despliega; las leyes generales de nuestra constitución resultan accesibles; y la historia sirve tanto a la filosofía moral como a la jurisprudencia" (Millar, [1803] 2006, p. 285).

Como acabamos de señalar, Millar, al modo de Smith, aboga por comenzar identificando, con base en su experiencia, aquellos hechos que considera veraces y relevantes, y a continuación los ordena, valiéndose para ello de una metodología lógico-deductiva. De este modo, por ejemplo, llega a afirmar que "cada arte práctico se erige sobre ciertos 
principios, descubiertos por la experiencia y la observación” (Millar, [1803] 2006, p. 729), que posteriormente, y mediante analogías y "cadenas de razonamientos" (Millar, [1803] 2006, p. 729), “dan lugar a diversas deducciones y conclusiones" (Millar, [1803] 2006, p. 729).

En cualquier caso, la forma de proceder de estos tres autores presenta una manifiesta afinidad con la doctrina metodológica expuesta previamente por David Hume. En este sentido, debemos señalar cómo este autor, por una parte, se refiere en múltiples ocasiones al sustrato experimental, propio de las reglas hipotético-deductivas, que respalda su doctrina; mientras que, por otra señala cómo las ciencias que mantienen una relación más estrecha e íntima con la naturaleza humana comprenden "casi todo aquello que nos puede importar" (Hume, [1739] 1896, p. xx). Entre estas últimas destaca la lógica, cuyo "fin único (...) consiste en explicar los principios y operaciones de nuestra facultad de razonamiento y la naturaleza de nuestras ideas" (Hume, [1739] 1896, p. xix); la moral y la crítica, que "reflexionan sobre nuestros gustos y sentimientos" (Hume, [1739] 1896, p. xix); y la política, que "atiende a cómo los hombres están unidos en sociedad y dependen entre sí” (Hume, [1739] 1896, p. xix).

\section{Agente individual}

Con respecto a la caracterización que estos autores llevan a cabo de los agentes individuales, lo primero que debemos señalar es que esta categoría presenta dos posibles vías de aproximación: la formal, basada en la construcción por parte del investigador de modelos pretendidamente no realistas; y la material, que atiende a su efectiva acción en el mundo. Dada la apuesta que estos autores realizan por cimentar sus investigaciones en la observación, no puede sorprendernos que se decanten por la segunda. Así, una vez dirimida esta cuestión, atendemos a dos de sus características más destacadas. Por una parte, comprobamos si estos autores defienden la tesis de que dichos agentes se mueven por criterios estrictamente egoístas; y, por otra, si consideran que disfrutan una ilimitada capacidad intelectual.

Nuestra hipótesis con respecto al primer rasgo consiste en afirmar que tanto Smith como Ferguson o Millar defienden que hay que buscar la motivación de los agentes no en el egoísmo propiamente dicho, sino en el interés propio. El antecedente teórico directo más notorio en este sentido remite de nuevo a la obra de David Hume. Este autor rechaza la posibilidad de atribuir al egoísmo una excesiva capacidad explicativa de la conducta humana. Sostiene que, pese a que "es raro encontrar a un hombre que ame a otra persona más que a sí mismo, es igualmente extraño encontrar una persona en la cual la suma de todos sus afectos generosos no supere a los egoístas [selfish]" (Hume, [1739] 1896, p. 487). 
En cualquier caso, antes de continuar debemos diferenciar entre agentes egoístas y agentes que demuestran interés propio. En el idioma inglés se distingue claramente entre selfish (egoísmo) -que remite, según el Diccionario de Oxford, a una "carencia de consideración hacia otras personas" a la hora de buscar los propios fines-y self-interest (interés propio) -que alude al "interés personal o ventaja de uno mismo, especialmente cuando se persigue sin tener en consideración a los demás"-. Es decir, la diferencia entre ambos conceptos radica en la necesaria, o no, toma en consideración del interés de otros a la hora perseguir determinados objetivos. Mientras que el egoísta nunca lo tiene en cuenta, el agente que demuestra interés propio puede incorporarlo.

Así, Adam Smith comienza su Teoría de los sentimientos morales afirmando, en la misma línea que Hume, que:

Por más que el hombre tenga rasgos egoístas [selfish], existen evidentemente en la naturaleza principios que le interesan en la suerte de los otros y que hacen que la felicidad de ellos le sea necesaria por más que no derive nada de esto, salvo el placer de poder contemplarla. (Smith, [1759] 1853, p. 3).

Como podemos comprobar, nos encontramos ante agentes que no se mueven exclusivamente por principios egoístas. Sin embargo, puede alegarse que esta es la postura que Smith defiende en La teoría de los sentimientos morales ([1759] 1853), y que con motivo de la publicación de La riqueza de las naciones ([1776] 1904) cambia de opinión. No es así. En esta última obra, Smith incluye tres referencias al término selfish y todas ellas presentan un carácter manifiestamente peyorativo. En la primera, reprueba el consumo ostentoso frente al consumo de recursos duraderos, por demostrar cierta disposición egoísta del agente y producir menos riqueza (Smith, [1776] 1904, p. 331 I). En la segunda, critica el carácter egoísta de los ciudadanos de aquellas naciones que prosperan gracias a la labor de mercaderes y manufactureros (Smith, [1776] 1904, p. 167 II). Y en la tercera, afirma que quizá no haya ningún placer egoísta tan frívolo que no haya arruinado en alguna ocasión incluso a las personas más sensatas (Smith, [1776] 1904, p. 392 II).

Una cita de Smith a la que puede acudirse como contraejemplo de nuestro argumento es la siguiente:

No es de la benevolencia del carnicero, del cervecero o del panadero que esperamos nuestra cena, sino de sus miras al interés propio [own interest]. Nos dirigimos, no a su humanidad, sino a su amor propio [self-love], y nunca les hablamos de nuestras necesidades, sino de sus ventajas. (Smith, [1776] 1904, p. 16 I).

En realidad, como podemos comprobar, Smith no se remite al egoísmo del carnicero, del cervecero o del panadero, sino a su propio interés (own interest) y amor propio (selflove). Por cierto, este segundo concepto alude a la "atención por el propio bienestar y felicidad de uno mismo". Existe, sin embargo, una clara diferencia, como ya hemos 
expuesto, entre considerar que un individuo es egoísta y que manifiesta interés o amor propio.

Además, la obra de Smith nos ofrece otro elemento que demuestra el error que supone considerar su caracterización de los agentes individuales como estrictamente egoístas: la sympathy (empatía). Este término, de nuevo según el Diccionario de Oxford, remite a "los sentimientos de pena y dolor por la desgracia de otra persona". No obstante, y pese a lo que pueda pensarse, este autor se apresura a aclarar que dicha aflicción no constituye una forma concreta de interés propio. La razón estriba en que, pese a que se pueda decir que "cuando yo me compadezco de tu dolor o indignación en cierto sentido mi emoción se basa en mi propio interés [self-love], (...) no se supone que este cambio imaginario suceda realmente en mi propia persona" (Smith, [1759] 1853, p. 465).

Por su parte, Adam Ferguson aboga por una doctrina muy similar a la de Smith al señalar cómo el lenguaje común establece una clara diferencia entre benevolencia y propio interés (o incluso egoísmo). Ciertas personas "han encontrado que la benevolencia no es más que una especie de amor propio [self-love]" (Ferguson, [1767] 1782, p. 23). Sin embargo, "es imposible vivir y actuar con los hombres sin emplear nombres diferentes para distinguir lo humano de lo cruel, y al benevolente del egoísta” (Ferguson, [1767] 1782 , p. 23).

No obstante, no debemos perder de vista cómo esta dicotomía que establece Ferguson entre egoísmo y benevolencia es parcialmente rebatida por Millar. Este autor comienza señalando cómo, efectivamente, las acciones virtuosas tienden a producir felicidad. Pero, a continuación, diferencia entre aquellas que favorecen "el bien del propio individuo [a man's own good]" (Millar, [1803] 2006, p. 791) y aquellas que "promueven el bien de otros" (Millar, [1803] 2006, p. 791). Mientras que las primeras hacen aflorar "sentimientos benévolos que satisfacen la felicidad de la persona que las realiza" (Millar, [1803] 2006, p. 791), las segundas "gratifican los sentimientos egoístas [selfish] del espectador" (Millar, [1803] 2006, p. 791), ya que este, según él, se considera como potencialmente incluido en la esfera de dicha acción. Así, según Millar, las acciones que promueven el bien de otros remiten, en última instancia, al egoísmo del observador.

Por otro lado, Millar, como Smith, también da entrada al concepto de empatía. En cualquier caso, a este autor no se le escapa cómo las personas que mantienen algún tipo de relación, pese a que "a menudo están vinculados por lazos de empatía [sympathy]" (Millar, [1803] 2006, p. 730), también pueden "discutir y pelear, e incluso producirse lesiones" (Millar, [1803] 2006, p. 730), dadas sus "pasiones e intereses contrapuestos" (Millar, [1803] 2006, p. 730).

De cualquier modo, no debemos confundir en la obra de Millar el interés propio con el desarrollo de conductas egoístas. En este sentido, alerta contra estas últimas al señalar 
cómo "la búsqueda de la riqueza (...) contribuye a dispersar las semillas de la envidia y el egoísmo, así como el hábito al lujo y la voluptuosidad" (Millar, [1803] 2006, p. 779), actitudes, todas ellas, que tienden a dar lugar a "producciones funestas" (Millar, [1803] 2006, p. 779).

El segundo elemento que define la caracterización que estos autores llevan a cabo de los agentes individuales remite al énfasis que imprimen en la naturaleza limitada del conocimiento del que disponen. Así, Hume (1826, p. 93) afirma de forma inequívoca que "el entendimiento humano queda infinitamente lejos de la sabiduría perfecta". La más clara manifestación de esta doctrina reside en su negación de una pretendida desvinculación de la racionalidad humana del mundo. No existe tal cosa como una racionalidad objetiva, externa a los individuos y sus pasiones: "Cuando hablamos del enfrentamiento 'entre pasión y razón', no nos expresamos correctamente. La razón es, y solo debe ser, esclava de las pasiones; el único trabajo que puede pretender hacer es servirlas y obedecerlas" (Hume, [1739] 1896, p. 415). En una línea muy similar se manifiesta Ferguson ([1767] 1782, pp. 290-291) al proclamar que, "bajo la supuesta desventaja de un conocimiento limitado (...), el simple poeta disfruta de impresiones que más que compensan el defecto de su habilidad".

Por su parte, Millar opta por destacar cómo la reducida capacidad de juicio de los agentes favorece el proceso de división del trabajo y del conocimiento, y afirma: "Dadas las limitadas capacidades, tanto de la mente como del cuerpo, los esfuerzos individuales resultarán más (...) exitosos cuando se centren en una sola finalidad que cuando se dispersen" (Millar, [1803] 2006, p. 731).

Finalmente, Smith hace descansar precisamente en esta limitada capacidad intelectual de los agentes su alegato en favor de mantener una actitud cautelosa frente a las consecuencias a las que pueden dar lugar los razonamientos erróneos:

La administración del gran sistema del universo (...) es el negocio de Dios y no de los hombres. A estos se les ha dado un departamento mucho más humilde, aunque más adecuado a la debilidad de sus poderes y a la cortedad de su comprensión: el cuidado de su propia felicidad, la de su familia, la de sus amigos y la de su localidad. (Smith, [1759] 1853, p. 348).

\section{Organizaciones}

Si nos aproximamos a las organizaciones como agentes corporados, debemos tener muy presente que estas se enfrentan con dos tipos de restricciones. En el ámbito interno, han de lidiar con problemas derivados de la necesaria coordinación de las acciones individuales; en el ámbito externo, han de ser capaces de operativizar la acción colectiva. Por lo tanto, a la hora de comparar la doctrina organizacional defendida por Adam Smith 
con la preconizada por los pioneros de la sociología británica, debemos atender a estos dos ámbitos diferenciados.

Así, debemos comenzar señalando cómo las reflexiones que estos autores llevan a cabo respecto a la esfera intraorganizativa se caracterizan por centrarse en el análisis del proceso de división del trabajo y por su ambivalente valoración al respecto. En este sentido, Adam Smith dedica el primer capítulo de La riqueza de las naciones a analizar dicho fenómeno y parte de una posición fundamentalmente optimista que destaca su papel como mecanismo de aumento de la riqueza: "Es la gran multiplicación de las producciones de todas las artes, como consecuencia de la división del trabajo, la que ocasiona, en una sociedad bien gobernada, esa opulencia universal que se extiende a los rangos más bajos de la población" (Smith, [1776] 1904, p. 12 I), una doctrina que encuentra su antecedente más inmediato en la afirmación de Hume de que, cuando una persona trabaja solo para sí misma, su limitada capacidad le imposibilita ejecutar labores complejas y hace que se encuentre a expensas del azar. De este modo, según Hume ([1739] 1896, p. 485), es gracias a la "partición de empleos" que "nuestra habilidad se incrementa; y debido al auxilio mutuo, se reduce nuestra exposición a hechos fortuitos o accidentes" (Hume, [1739] 1896, p. 485).

Sin embargo, Adam Smith no obvia los efectos negativos que el proceso de división del trabajo presenta. Así, defiende que, según este avanza,

el empleo de la mayor parte de los que viven del trabajo (...) se reduce a unas pocas operaciones muy sencillas (...) El hombre cuya vida discurre en la realización de operaciones sencillas cuyos efectos también son quizá siempre iguales, o casi, no tiene ocasión de ejercer su entendimiento o ejercitar su creatividad para resolver unas dificultades que nunca ocurren. Como es natural, pierde, por lo tanto, el hábito de tal esfuerzo, y por lo general se vuelve tan estúpido e ignorante como es posible que un ser humano llegue a ser. (Smith, [1776] 1904, p. 267 II).

De hecho, Smith continúa afirmando que estas personas son incapaces de disfrutar de una conversación racional, mostrar sentimientos nobles, justos y generosos, o defender a su país en la guerra. Según este autor, por lo tanto, el coste de la división del trabajo se plasma en una reducción de las virtudes intelectuales y cívicas de los ciudadanos.

Por su parte, Adam Ferguson, frente a la posición defendida por Smith - y que podemos calificar como fundamentalmente optimista, pero con reparos- opone una perspectiva pesimista, pero que no niega su papel a la hora de explicar el crecimiento económico. Su crítica parte de una concepción estoica del individuo que destaca el riesgo de reducirlo a la condición de mera pieza de una máquina:

La ignorancia es la madre de la industria y la superstición. La reflexión y el talento imaginativo pueden inducir a error, pero el hábito de mover el pie o la mano nada tiene que ver ni con la una ni con el otro. Por eso, donde más prosperan las manufacturas es allí donde se 
deja menos margen al espíritu, hasta el punto de que el taller podría ser definido como una máquina cuyas piezas son hombres. (Ferguson, [1767] 1782, p. 305).

Es decir, la doctrina estoica por la que aboga Ferguson no le permite contemplar con buenos ojos el creciente proceso de división del trabajo, aunque reconoce su utilidad en el ámbito estrictamente económico. Según este autor, los individuos deben desarrollar una serie de virtudes ciudadanas que mantengan los lazos sociales, ya que, en caso contrario, la sociedad corre el riesgo de colapsar:

Los miembros de una comunidad pueden, de esta manera, (...) perder el sentido de sus conexiones, salvo los de parentesco o vecindad, y no compartir los asuntos comunes necesarios para realizar transacciones, salvo los del comercio. Conexiones, en efecto, o transacciones, en las que la probidad y la amistad todavía pueden tener lugar, pero en las que el espíritu nacional (...) no puede ser ejercido. (Ferguson, [1767] 1782, p. 367).

Como podemos comprobar, Smith y Ferguson consideran que la división del trabajo produce tanto un aumento de la riqueza como un riesgo para las virtudes intelectuales y cívicas de los individuos. La diferencia entre ambos autores radica en la importancia relativa que cada uno de ellos atribuye a estos dos factores. Mientras que Smith se decanta por destacar el crecimiento económico a que da lugar, Ferguson se centra en criticar sus perniciosas consecuencias sociales.

En esta misma línea se pronuncia John Millar. Este autor llama la atención sobre el hecho de que la división del trabajo manifiesta una doble tendencia: por una parte, "tiende a mejorar" (Millar, [1803] 2006, p. 731) las artes y las ciencias; y, por otra, muestra "el efecto contrario sobre las cualidades personales de aquellos que desempeñan tales funciones" (Millar, [1803] 2006, p. 732). Así, "su continua atención a los objetos de su profesión (...) presenta una poderosa disposición a hacerlos ignorantes y estúpidos" (Millar, [1803] 2006, p. 732). Dos adjetivos de los que, como ya hemos visto, se vale previamente el propio Adam Smith.

Por otro lado, en la esfera interorganizativa, Smith alerta sobre el riesgo que supone el surgimiento de monopolios. Pero esta crítica no se restringe a las alianzas empresariales, sirve también para censurar determinadas prácticas sindicales:

Los dueños de las empresas también, a veces, llegan a acuerdos particulares para hundir los salarios del trabajo incluso por debajo de esta tasa. (...) Este tipo de acuerdos, sin embargo, con frecuencia no se consolidan debido a un acuerdo contrario por parte de los obreros que se defienden. Estos últimos también, a veces, demasiadas, sin ninguna provocación de este tipo, llegan a acuerdos por su propia voluntad para elevar el precio de su trabajo. (Smith, [1776] 1904, p. 69 I).

En esta misma línea, Millar ([1803] 2006, pp. 714-715) se manifiesta contrario al establecimiento de monopolios estatales por tender estos "a disminuir la cantidad, así como degradar la calidad de los productos implicados”. Pero, al mismo tiempo, también abre 
la puerta a dichas prácticas "en la infancia del comercio" (Millar, [1803] 2006, p. 714), ya que, según él, "tales regulaciones tal vez fueron necesarias para fomentar empresas nuevas y peligrosas” (Millar, [1803] 2006, p. 714).

\section{Instituciones}

La última categoría a la que atendemos remite a las instituciones. Definimos estas como "reglas constitutivas" (Searle, [1995] 1997, p. 45), es decir, como pautas que "no solo regulan, sino que crean la posibilidad misma de ciertas actividades" (Searle, [1995] 1997, p. 45). Así, apoyándonos en Hayek (1946), analizamos cuatro de sus características principales. En primer lugar, nos fijamos en su carácter general, tanto a nivel diacrónico -remitiéndonos a su evolución histórica- como sincrónico -comprobando si se incluyen instituciones formales e informales-. En segundo lugar, verificamos si se defiende una concepción natural de aquellas que remita su génesis y su lógica de funcionamiento a la propia condición humana. En tercer lugar, aludimos a su surgimiento espontáneo como producto no deliberado de la acción. Y finalmente nos referimos a las posibles relaciones conflictivas que pueden establecerse entre ellas, es decir, a la existencia de contradicciones potenciales entre las funciones que los agentes les atribuyen.

Con respecto al carácter general de las instituciones, hemos de comenzar destacando cómo Adam Smith y los pioneros de la sociología británica abogan por atender tanto aquellas de perfil formal como las de perfil no formal. En este sentido, podemos remitirnos a la manera en que Millar vincula el progreso histórico del Estado (formal) al de las familias (informal); cómo Ferguson se remite asiduamente tanto a los modales (informal) como al establecimiento de los Estados (formal); o, finalmente, al modo en que Adam Smith analiza las diferentes costumbres de las naciones (informal) y las políticas públicas de los Estados (formal).

Por otra parte, todos estos pensadores coinciden en señalar que dichas instituciones evolucionan a lo largo de la historia. En este sentido, el ejemplo paradigmático remite a la obra de Millar ([1771] 2006) Observaciones sobre la distinción de rangos en la sociedad. Como acabamos de señalar, en este texto el autor pretende poner en relación el surgimiento y el progreso histórico del Estado con las diferentes formas históricas que van adoptando las estructuras de parentesco. Así, defiende que este se origina en comunidades organizadas en familias que posteriormente se agrupan formando clanes, que, a su vez, desembocan en la autoridad real.

Por otro lado, y como hemos señalado previamente al tratar de las fases en las que estos autores dividen la historia humana, dicha secuencia avanza de la "rudeza" a las "maneras civilizadas". Sin embargo, la caracterización que estos autores llevan a cabo de tal 
proceso presenta dos particularidades. Por una parte, puede verse frenado, y, por otra, es susceptible de presentar formas múltiples. En este sentido, por ejemplo, en relación con la libertad, Ferguson ([1767] 1782, p. 214) afirma que esta, "en su ascenso, y en medio de las interrupciones que los intereses opuestos provocan, puede resultar permanente o transitoria; y su constitución puede presentar formas tan variadas como la combinación ocasional de sus partes permita".

También en esta misma línea se pronuncia Millar ([1771] 2006, p. 65) al señalar que "diversas causas accidentales" pueden contribuir a "acelerar o retardar este avance [de la libertad] en los diferentes países” (Millar, [1771] 2006, p. 65).

Sin embargo, cuando atendemos a las reflexiones de David Hume al respecto, observamos una peculiaridad. Este autor, pese a que defiende que las instituciones son generales por cuanto, por una parte, incluyen aquellas de carácter formal y no formal, y, por otra, presentan un carácter histórico (Hume, [1777] 1987, p. 28), también se decanta por introducir un modelo cíclico de auge y declive del todo ajeno al esquema secuencial y progresivo por el que abogan estos autores. Así, por ejemplo, al tratar sobre el progreso de las artes y de las ciencias, afirma que cuando estas "se aproximan de algún modo a la perfección, desde ese momento (...) necesariamente declinan. Rara vez, o ninguna, vuelven a florecer en esa nación" (Hume, [1777] 1987, p. 135).

El segundo rasgo de la teoría institucional que comparten todos estos autores remite a establecer su origen en la propia condición humana. Así, como ya hemos visto, los agentes individuales se caracterizan por su limitada capacidad intelectual, interés propio y empatía. Pero a esta forma de comprenderlos, Ferguson añade, además, la idea de que los razonamientos de los individuos pueden resultar, en ocasiones, contradictorios, escenario ante el cual emergen instituciones que les permiten mitigar los efectos perniciosos que pueden derivarse de tales circunstancias. Esta forma humanista de aproximarse al origen de las instituciones es la que explica que Smith ([1776] 1904, p. 15 I) haga descansar su comprensión de la división del trabajo en "una cierta propensión en la naturaleza humana (...) al transporte, trueque y cambio de una cosa por otra", o que Millar ([1803] 2006, p. 779) defienda que los "placeres sensuales [una característica de la condición humana] (...) se pueden conectar en muchos casos con los ejercicios de disposiciones sociales". Millar llega a ilustrar esta última tesis afirmando que "el afecto conyugal, cuando se unió al amor por los hijos, fue capaz (...) de cimentar las familias, y así sentó las bases de la sociedad política" (Millar, [1803] 2006, p. 765).

También en este punto, la posición teórica defendida por Hume resulta, aunque en términos generales coincidente, particularmente original. Si bien es cierto que este autor remite el origen de las instituciones a la propia condición humana, no lo es menos que también establece una posible salvedad: el gobierno. Así, por una parte, afirma que " $[\mathrm{t}]$ odas las instituciones humanas, y ninguna más que el gobierno, presentan una continua 
fluctuación" (Hume, [1777] 1987, pp. 494-495); es decir, considera al gobierno como una institución humana. Pero, por otra parte, también defiende que "la DEIDAD es el autor último de todo gobierno" (Hume, [1777] 1987, p. 466). ¿Cómo solventa Hume esta aparente contradicción? Apoyándose, por una parte, en el limitado conocimiento de los seres humanos; y, por otra, en el hecho de que "es imposible para la raza humana subsistir (...) sin la protección de un gobierno" (Hume, [1777] 1987, p. 466). Habida cuenta de estos dos factores, y dado que los gobiernos existen, concluye que "esta institución debe (...) haber sido prevista por ese Ser benéfico que procura el bien de todas sus criaturas" (Hume, [1777] 1987, pp. 466-467).

De cualquier modo, tanto Adam Smith como los pioneros de la sociología británica -y aquí reside el elemento más característico de su doctrina institucional- rechazan remitir el origen y la forma de las instituciones a actos deliberados de los individuos. El surgimiento y la evolución de las instituciones es, en la mayor parte de los casos, una consecuencia no prevista de la acción humana que da como resultado una mejora de las condiciones de vida de los individuos. Como afirma Adam Ferguson:

Cada paso y cada movimiento de la multitud, incluso en lo que se denomina la edad de la razón, se realiza con igual ceguera hacia el futuro. Las naciones se tropiezan con las inercias [establishments], que son en realidad el resultado de la acción humana, pero no la ejecución de un proyecto humano. (Ferguson, [1767] 1782, p. 205).

En este mismo sentido es en el que debemos comprender la tesis que defiende Millar de que el surgimiento de la libertad política puede ser comprendido como una consecuencia no prevista del enfrentamiento entre tiranos.

Por otro lado, Adam Smith ([1776] 1904, p. 421 I) también se manifiesta en esta misma línea al afirmar que los individuos, "persiguiendo su propio interés [own interest], frecuentemente promueven el de la sociedad con más eficiencia que si realmente intentaran promover el interés público". Así, y a modo de ejemplo, podemos atender al carácter institucional de la propia división del trabajo. Esta:

no es originalmente el efecto de cualquier sabiduría humana que prevé y pretende la opulencia general a la que da lugar. Es la consecuencia necesaria, aunque muy lenta y gradual, de una cierta propensión de la naturaleza humana que no tiene a la vista ninguna utilidad tan amplia. (Smith, [1776] 1904, p. 15 I).

Sin embargo, Hume ([1777] 1987, p. 54) se distancia de esta doctrina al defender que "LEGISLADORES y fundadores de Estados" transmiten deliberadamente "un sistema de leyes e instituciones para asegurar la paz, felicidad y libertad de las futuras generaciones" (Hume, [1777] 1987, p. 54). Este distanciamiento se confirma definitivamente al calificar este mismo autor tales hechos como "logros memorables" (Hume, [1777] 1987, p. 54) de dichos agentes. 
La última característica de la doctrina institucional de estos autores a la que atendemos es su carácter conflictivo. En este sentido, quizá el mejor ejemplo lo encontremos, precisamente, en el debate previamente presentado sobre las consecuencias en el ámbito económico y en el civil de la división del trabajo. Como recordamos, todos estos pensadores llaman la atención sobre cómo dicho fenómeno, si bien procura un innegable aumento del bienestar material, también se cobra un alto precio en términos de virtudes ciudadanas.

\section{Conclusiones}

A la vista de estos resultados, podemos concluir afirmando que, si bien, tanto Adam Smith como los pioneros de la sociología británica (Adam Ferguson y John Millar) priorizan el interés práctico de comprensión, también establecen una forma epistemológica híbrida de reglas hipotético-deductivas e histórico-hermenéuticas. Esta nueva configuración se traduce, en el ámbito metodológico, en una estrategia que consiste en partir de la identificación, con base es su experiencia -es decir, a la manera empírico-analítica-, de aquellos factores que consideran más relevantes, para posteriormente establecerlos como principios generales desde los cuales deducir conclusiones mediante procedimientos lógicos (de un modo lógico-deductivo).

Por otra parte, todos estos autores también parten de una caracterización de los agentes individuales de signo material (no formal) que enfatiza su limitado conocimiento y el hecho de que no se mueven exclusivamente por principios egoístas. Además, atienden a las ambivalentes consecuencias que el proceso de división del trabajo acarrea por cuanto supone un manifiesto incremento de la riqueza, pero también un riesgo para aquellas virtudes cívicas que consideran necesarias para el buen funcionamiento de la sociedad. Y, finalmente, consideran las instituciones como fenómenos generales que emergen de la propia condición humana, pero que, pese a ser el resultado de las acciones de los individuos, no deben comprenderse como productos intencionales de estos. Del mismo modo, contemplan la posibilidad de que tales instituciones entren en conflicto al presentar funciones contradictorias. En definitiva, esta investigación nos permite demostrar la fundamental concordancia teórica existente entre las aportaciones procedentes de Adam Smith y de los pioneros de la sociología británica: Adam Ferguson y John Millar.

Además, constatamos cómo muchos de los supuestos sobre los que estos autores edifican su sistema teórico encuentran su formulación original en la obra de David Hume y cómo gracias a ello podemos afirmar que nos encontramos ante un corpus teórico coherente. No debemos olvidar que este autor, figura central del pensamiento ilustrado escocés, mantiene una relación personal más o menos estrecha con todos ellos. Así, nombra a Adam Smith albacea de sus manuscritos (Mellizo, 1999, p. 8); a Adam Ferguson, 
compañero suyo en la Universidad de Edimburgo, le sustituye al frente de la biblioteca de la Facultad de Derecho; y como John Millar ([1803] 2006), a la sazón de alumno de Smith, redacta Una visión histórica del Gobierno inglés en forma de réplica a su Historia de Inglaterra (Hume, [1754-1761] 1842-1844).

\section{Referencias}

Barnes, H. E. (1917). Sociology before Comte: A summary of doctrines and an introduction to the literature. American fournal of Sociology, 23(2),174-247.

Benton, T. (1978). How many Sociologies? The Sociological Review, 26, 217-236. DOI: 10.1111/j.1467-954X.1978.tb00131.x

Brewer, J. D. (2007). Putting Adam Ferguson in his place. The British Fournal of Sociology, 58(1), 105-122. DOI: 10.1111/j.1468-4446.2007.00141.x

Bryson, G. (1939). Some eighteenth-century conceptions of society. The Sociological Review, 31,401-421.

Ferguson, A. ([1767] 1782). An essay on the history of civil society. Londres: T. Cadell.

Habermas, J. (1968). Conocimiento e interés. En: M. Jiménez (Ed.), Jürgen Habermas. Conocimiento e interés. Edmund Husserl. La filosofia en la crisis de la humanidad europea. Valencia: Universitat de Valencia.

Hayek, F. A. von. (1946). Individualism: True and false. En F. Hayek, Individualism and economic order. Chicago: The University of Chicago Press.

Hume, D. ([1739] 1896). Treatise of human nature. Oxford: Clarendon Press.

- ([1754-1761] 1842-1844). Historia de Inglaterra desde la invasión de fulio César hasta el fin del reinado de Facobo II (año de FC, 1689) (cuatro volúmenes). Barcelona: Imprenta de Francisco Oliva.

. ([1777] 1987). Essays moral, political, literary. Indianápolis: Liberty Fund. Recuperado de http://lf-oll.s3.amazonaws.com/titles/704/0059_Bk.pdf

. (1826). The philosophical works of David Hume. Including all the essays, and exhibiting the more important alterations and corrections in the successive editions by the author (cuatro volúmenes). Edimburgo: Adam Black y William Tait. 
Lamo de Espinosa, E. (2001). La sociología del siglo XX. REIS. Revista Española de Investigaciones Sociológicas, 96, 21-49. Recuperado de goo.gl/afRR5O

Lehmann, W. C. (1930). Adam Ferguson and the beginnings of modern sociology. Nueva York: Columbia University Press. . (1960). Fohn Millar of Glasgow 1735-1801: His life and thought and his contributions to sociological analysis. Cambridge: Cambridge University Press.

Mellizo, G. (1999). Prólogo. En D. Hume, Diálogos sobre la religión natural. Madrid: Alianza Editorial.

MacRae, D. G. (1969). Adam Ferguson. En The founding fathers of social science (pp. 17-26). Londres: Penguin Books.

Millar, J. ([1771] 2006). The origin of the distinction of ranks; or, an inquiry into the circumstances which give rise to influence and authority in the different members of society. Indianápolis: Liberty Fund. Recuperado de http://lf-oll.s3.amazonaws.com/titles/287/1342_LFeBk.pdf . ([1803] 2006). An historical view of the English government, from the settlement of the Saxons in Britain to the revolution in 1688. Indianápolis: Liberty Fund. Recuperado de http://lf-oll.s3.amazonaws.com/titles/1886/1365_LFeBk.pdf

Searle, J. R. ([1995] 1997). La construcción de la realidad social. Barcelona: Paidós.

Skinner, A. S. (1972). Adam Smith: Philosophy and science. Scottish fournal of Political Economy, 19(3), 307-319. DOI: 10.1111/j.1467-9485.1972.tb00527.x

(1976). Adam Smith: The development of a system. Scottish fournal of Political Economy, 23(2), 111-132. DOI: 10.1111/j.1467-9485.1976.tb00769.x

Small, A. W. (1907). Adam Smith and modern sociology: A Study in the methodology of the social sciences. Chicago: Chicago University Press.

Smith, A. ([1759] 1853). The theory of moral sentiments; or, an essay towards an analysis of the principles by which men naturally judge concerning the conduct and character, first of their neighbors, and afterwards of themselves. To which is added, a dissertation on the origins of languages. Londres: Henry G. Bohn.

. ([1762-63] 1896). Lectures on justice, police, revenue and arms. Oxford: Clarendon Press.

. ([1776] 1904). An inquiry into the nature and causes of the wealth of nations. (Vol. I y II). Londres: Methuen. 
. (1795). Essays on philosophical subject by the late Adam Smith, LL. D. Londres y Edimburgo: T. Cadell Jun. y W. Davies (Successors to Mr. Cadell).

Stewart, D. (1795). Account of the life and writings of Adam Smith LL.D. En Essays on philosophical subject by the late Adam Smith, LL.D. Londres y Edimburgo: T. Cadell Jun. y W. Davies (Successors to Mr. Cadell).

Swingewood, A. (1970). Origins of sociology: The case of the Scottish enlightenment. The British fournal of Sociology, 21(2), 164-180. DOI: 10.2307/588406 